\title{
Determination of water and salt transport parameters of porous materials using methods of inverse modelling
}

\author{
L. Fiala, Z. Pavlík, M. Pavlíková \& R. Černý \\ Department of Building Materials, Faculty of Civil Engineering, \\ Czech Technical University in Prague, Czech Republic
}

\begin{abstract}
A computational assessment of water and salt transport parameters describing the coupled moisture and chloride transport in porous media is presented in this paper. The experimentally determined moisture profiles, chloride concentration profiles and chloride binding isotherm are subjected to inverse analysis where three different modes of salt solution transport are assumed. On the basis of this analysis, moisture dependent moisture diffusivity and salt concentration dependent chloride diffusion coefficient are calculated. The obtained results can find use in computational modelling of salt transport in porous building materials which is currently a very important topic, particularly from the point of view of durability of building materials and service life of building structures exposed to salt attack.
\end{abstract}

Keywords: moisture diffusivity, salt diffusion coefficient, chloride binding isotherm.

\section{Introduction}

The durability and service life assessment of building materials and structures due to the effect of salts can be done most effectively by means of mathematical and computational modelling. In this way, the time development of water and salt concentration fields can be obtained which is crucial for prediction of possible future damage related to the salt crystallization and efflorescence. However, the accuracy of simulated water and salt concentration fields critically depends on the availability of all input parameters. 
There are two types of input parameters of computational models of water and salt transport which have to be known in advance. The first are initial and boundary conditions which can be obtained relatively easily using on site analysis of water and salt concentration fields in the walls and in subsoil, and meteorological data for relative humidity, temperature and rain. The second type of input parameters are water and salt transport and storage parameters of building materials which appear in water and salt mass balance equations.

In this work, the main attention is paid to the determination of water and salt transport parameters on the basis of inverse analysis of experimentally determined salt concentration and moisture profiles.

\section{Mathematical models of salt transport}

Identification of parameters describing the salt transport is based on the assumed mode of this transport. In this work, three different approaches for determination of salt transport parameters were used.

At first, simple Fick's diffusion equation with constant salt diffusion coefficient and constant initial and boundary conditions was employed. In this model, only diffusion transport of salts is taken into account. The Fick's diffusion equation has a very simple mathematical solution in the following form $[1,2]$

$$
C(x, t)=C_{0}\left[1-\operatorname{erf}\left(\frac{x}{2 \sqrt{D t}}\right)\right],
$$

where $C\left[\mathrm{~kg} / \mathrm{m}^{3}\right]$ is salt concentration, $D\left[\mathrm{~m}^{2} \mathrm{~s}^{-1}\right]$ salt diffusion coefficient, $C_{o}$ $\left[\mathrm{kg} / \mathrm{m}^{3}\right]$ salt concentration on the end of the sample exposed to the salt solution, $x$ $[\mathrm{m}]$ the distance from the exposed end of the sample, $t$ [s] time. The main disadvantage of this model is the assumption of constant diffusion coefficient and neglect of the influence of water transport on salt transport. However, this model is very simple; so it is frequently used by many authors for some rough estimation of the salt transport velocity.

The second type of applied model was based on diffusion mechanism of salt solution transport as well. Compared to Fick's diffusion equation, the dependence of salt diffusion coefficient $D$ on salt concentration $C$ was involved,

$$
\frac{\partial C}{\partial t}=\operatorname{div}(D(C) \operatorname{grad} C),
$$

where $C[\mathrm{~kg} / \mathrm{kg}]$ is salt concentration in $\mathrm{kg}$ per $\mathrm{kg}$ of the dry porous body, $D\left[\mathrm{~m}^{2} \mathrm{~s}^{-1}\right]$ the apparent salt diffusion coefficient. In this way the salt solution transport is formally described by the same parabolic equation with the same boundary and initial conditions usually used for description of water transport. Therefore, the calculation of concentration-dependent diffusion coefficients from the measured salt concentration profiles could be done using basically the same 
inverse methods as those for the determination of moisture-dependent moisture diffusivity or temperature-dependent thermal conductivity. In this paper, this type of model was employed for determination of both $D(C)$ and $\kappa(w)$ functions. In the inverse analysis, the Matano method which is the most frequently used method in the inverse analysis of moisture profiles was employed [3, 4]. Application of Matano method gives the following final solution for salt diffusion coefficient

$$
D\left(C_{0}\right)=\frac{1}{2 t_{0}\left(\frac{d C}{d z}\right)_{z=z_{0}}} \int_{z_{0}}^{\infty} z \frac{d C}{d z} d z,
$$

and for moisture diffusivity

$$
\kappa\left(w_{0}\right)=\frac{1}{2 t_{0}\left(\frac{d \kappa}{d z}\right)_{z=z_{0}}} \int_{z_{0}}^{\infty} z \frac{d \kappa}{d z} d z,
$$

where $C_{0}=C\left(z_{0}, t_{0}\right)$ is salt concentration in the position $z_{0}$ and time $t_{0}, w_{0}=$ $w\left(z_{0}, t_{0}\right)$ the corresponding moisture content and $\mathrm{z}$ space variable. The integral in Eqs. (3) and (4) is solved by common numerical methods, such as Simpson's rule.

The most advanced model applied in this paper for identification of the $D(C)$ function was the Bear and Bachmat diffusion-advection model $[5,6]$ taking into account (in addition to salt diffusion in the liquid phase) the influence of moisture flow on salt transport and also the effect of bound salt on pore walls. This model employs for description of coupled salt and water transport the system of parabolic equations

$$
\begin{gathered}
\frac{\partial\left(w C_{f}\right)}{\partial t}=\frac{\partial}{\partial x}\left(w D \frac{\partial C_{f}}{\partial x}\right)+\frac{\partial}{\partial x}\left(C_{f} \kappa \frac{\partial w}{\partial x}\right)-\frac{\partial C_{b}}{\partial t}, \\
\frac{\partial w}{\partial t}=\frac{\partial}{\partial x}\left(\kappa \frac{\partial w}{\partial x}\right),
\end{gathered}
$$

where $C_{f}\left[\mathrm{kgm}^{-3}\right]$ is the concentration of free salts in water, $C_{b}\left[\mathrm{kgm}^{-3}\right]$ the concentration of bound salts in the whole porous body and $w\left[\mathrm{~m}^{3} \mathrm{~m}^{-3}\right]$ the volumetric moisture content.

This system of equations can be subjected to an inverse analysis in a similar way as for one parabolic equation, provided the initial and boundary conditions are simple enough, and the material parameters $D$ and $\kappa$. can be identified as functions of water content and salt concentration. The simplest possibility of such an inverse analysis is again an extension of the Boltzmann-Matano treatment under the same assumptions of constant initial conditions and Dirichlet boundary conditions on both ends of the specimen for both moisture content and salt concentration where one of the Dirichlet boundary conditions is equal to the initial condition. 


\section{Experimental}

In the experimental part of this work, ceramic brick having bulk density $\rho_{b}=2044 \mathrm{kgm}^{-3}$ and matrix density $\rho_{\text {mat }}=2666 \mathrm{kgm}^{-3}$ was studied. The arrangement of the experiment for determination of moisture and salt concentration profiles was analogous to standard water suction experiments. The samples with the dimensions of $20 \times 40 \times 160 \mathrm{~mm}$ were first dried at $80^{\circ} \mathrm{C}$ and 0.1 mbar at vacuum drier and water and vapour-proof insulated by epoxy resin on all lateral sides. Then, they were exposed by their $40 \times 20 \mathrm{~mm}$ face to the penetrating $1 \mathrm{M}-\mathrm{NaCl}$ solution $\left(\rho_{I M-\mathrm{NaCl}}=1041 \mathrm{kgm}^{-3}\right.$ at $\left.21{ }^{\circ} \mathrm{C}\right)$. Duration of the experiment was 30,60 and 120 minutes for three different groups of samples. After this time, the samples were cut into 8 pieces and in each piece water content and chloride concentration were measured. Moisture content was determined by the gravimetric method using weighing the moist and dried specimens. In the determination of chloride concentration, the particular samples were after drying first ground by a vibration mill so that grains smaller than $0.063 \mathrm{~mm}$ were obtained. Then the ground samples were overflowed by $80{ }^{\circ} \mathrm{C}$ warm distilled water and leached. The chloride contents in particular leaches were determined using an ion selective electrode.

For the determination of ion binding isotherm as the main salt storage parameter, a modification of the Tang and Nilsson adsorption method [7] was chosen. The modification of the Tang and Nilsson method applied in our measurements consisted in using the specimens of more realistic dimensions $(40 / 40 / 10 \mathrm{~mm})$ instead of crushed specimens. The main reason for this was that the original method presents certain idealization of the binding problem assuming that salts can get in direct contact with every small grain of the measured material. However, in a real specimen the interior pore surface where the material is accessible to the salts is certainly smaller than the total surface of a crushed specimen. The salt binding capacity can be then affected by many other factors such as the change in the porous structure and pore distribution due to the presence of various admixtures, etc. Therefore, the result obtained by the adsorption method in its original form can be considered as a certain upper limit to the real salt binding capacity.

On the basis of the measured ion binding isotherm of $\mathrm{NaCl}, C_{b}=f\left(C_{f}\right)$, the profiles of bound and free chlorides were determined.

\section{Results and discussion}

The experimentally determined moisture and salt concentration profiles are presented in Figs. 1 and 2. For approximation of measured data which is necessary for computer data processing, the linear filtration method was used. In Figs. 1 and 2, the smoothed data for approximation parameter $\alpha=1.9$ are shown as well to illustrate the quality of smoothing procedure.

The chloride binding isotherm measured by modified Tang and Nilsson adsorption method is shown in Fig. 3. The obtained binding isotherm gives clear evidence about the high chloride binding capacity of the studied material. 


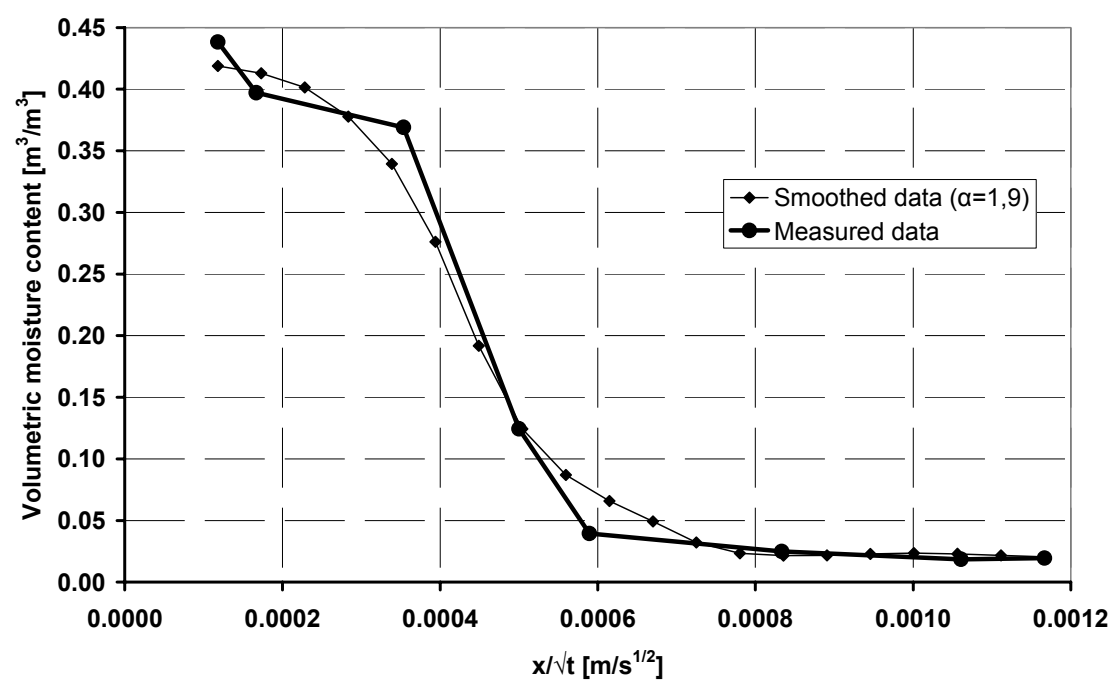

Figure 1: Volumetric moisture content for $1 \mathrm{M}-\mathrm{NaCl}$ solution transport.

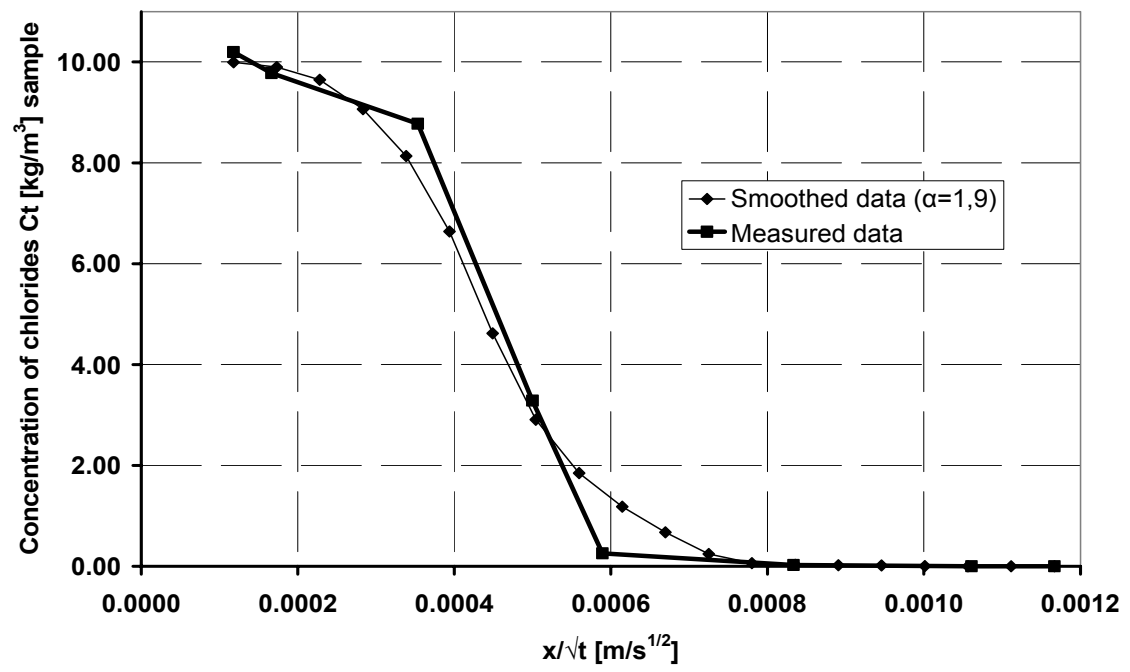

Figure 2: Total salt concentration.

Fig. 4 shows the moisture diffusivity of investigated ceramic brick as a function of moisture content calculated using the inverse analysis described before. We can see that the moisture diffusivity values for the penetration of pure distilled water were systematically higher than those obtained for salt solution penetration. This result is not surprising and is in accordance with higher 
viscosity of salt solution as compared to water. The upper curve of moisture diffusivity function corresponds to the inverse analysis neglecting the effect of diffusion (dispersion) in salt solution transport. The results give evidence that the diffusion (dispersion) mechanism is quite important in the solution transport.

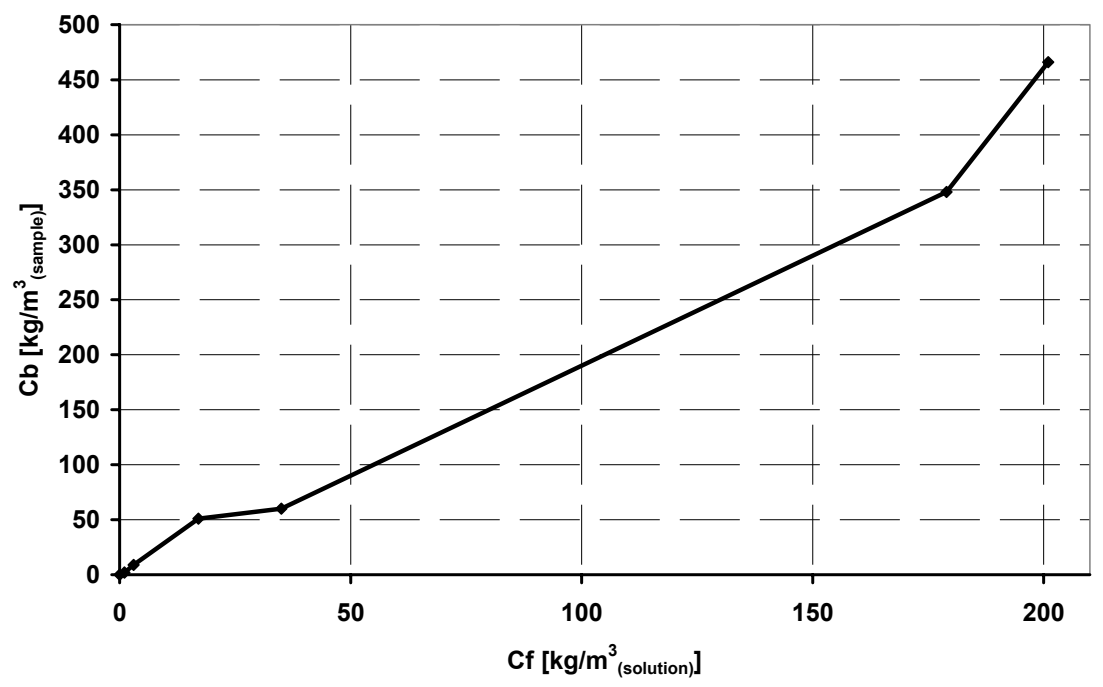

Figure 3: Chloride binding isotherm of ceramic brick.

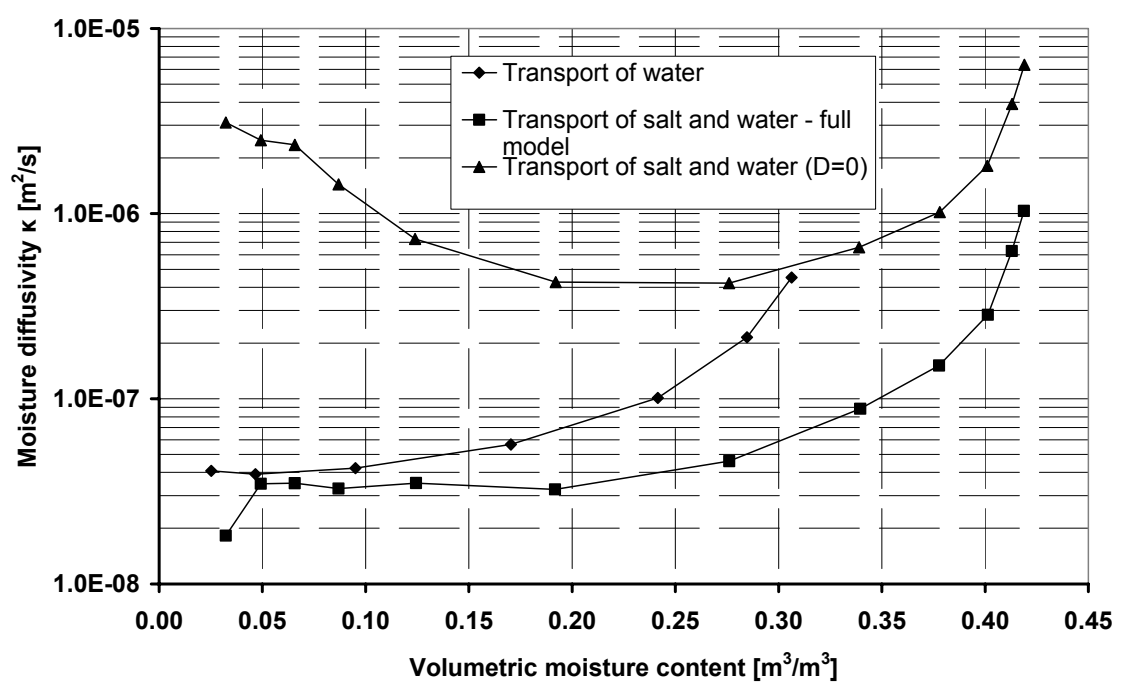

Figure 4: $\quad$ Moisture diffusivity of ceramic brick. 
The chloride diffusion coefficient of ceramic brick calculated in dependence on free ion concentration by three different methods of inverse analysis is shown in Fig. 5. We can see that from the quantitative point of view, the calculated dispersion coefficient is quite high, about three orders of magnitude higher than the diffusion coefficients of most ions in free water. Therefore, the common diffusion mechanism was probably not the only driving force for the chloride transport within the liquid phase and some other driving forces were taking place here. The acceleration of chloride transport can be attributed most easily to surface diffusion on pore walls or to some preferential paths of salt solution transport. It should be noted, however, that this is a formal explanation only and an exact physico-chemical analysis is still needed. The diffusion coefficient determined using the diffusion-advection concept was one to two orders of magnitude higher than both diffusion-model based coefficients. This means that the advection mechanism was quite significant in the process of salt transport. This feature we have already observed for other studied common building materials as sandstone or calcium silicate.

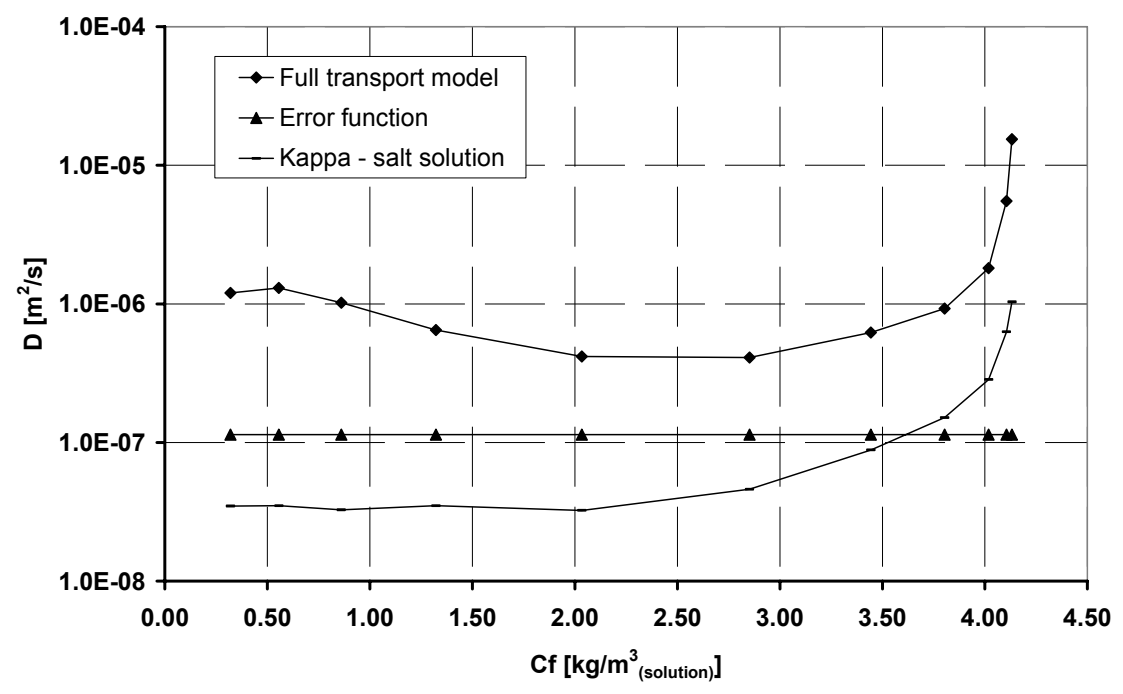

Figure 5: Chloride diffusion coefficient of ceramic brick.

\section{Conclusions}

Theoretical and experimental studies of coupled water and salt transport belong still to actual topics in describing transport phenomena in building materials because such descriptions are not yet very frequent in building science. In this paper, an attempt towards better understanding of mechanisms driving the coupled water and chloride transport in porous media was done. This analysis has clearly shown that description of salt transport in building materials should always be done in a combination with water transport. It also confirmed that an 
application of ion binding isotherms in mathematical models of water and salt transport is an unavoidable condition for a realistic description of processes taking place in the porous medium. Neglecting the effects of water transport in the porous material and ion absorption on the pore walls that is common in the simplest diffusion models using solely salt diffusion coefficients can lead to ambiguous results in inverse analysis of salt concentration profiles, and consequently to a departure from the reality. If such oversimplified models are used anyway, their application should always be done for relative purposes only and bearing in mind that some very important factors were neglected.

This paper contributed to both theoretical and practical description of coupled water and salt transport in porous materials. From the practical point of view, two main parameters describing the capability of ceramic brick to transport salt and moisture were accessed. From the point of view of explanation of mechanisms of coupled water and salt transport in porous materials, the presented experiments and calculations revealed the necessity to implement some other effects (e.g., surface diffusion, preferential transport paths) into the diffusion-advection model.

\section{Acknowledgement}

This research was supported by the Ministry of Education, Youth and Sports of Czech Republic, under grant No. MSM: 6840770031.

\section{References}

[1] Carslaw, H.S. \& Jaeger, J.C., Conduction of Heat in Solids, Clarendon Press: Oxford, 1959.

[2] Weyers, R.E., Service life model for concrete structures in chloride laden environments. ACI Materials Journal, 95, pp. 445-453, 1998.

[3] Matano, C., On the relation between the diffusion coefficient and concentration of solid metals. Jap. J. Phys., 8, pp. 109-115, 1933.

[4] Černý, R., Pavlík, Z. \& Rovnaníková, P., Experimental analysis of coupled water and chloride transport in cement mortar. Cement and Concrete Composites, 26, pp. 705-715, 2004.

[5] Bear, J. \& Bachmat, Y., Introduction to Modelling of Transport Phenomena in Porous Media, Vol 4, Kluwer: Dordrecht, 1990.

[6] Pel, L., Kopinga, K., \& Kaasschieter, E. F., Saline absorption in calciumsilicate brick observed by NMR scanning. J. Phys. D: Appl. Phys, 33, pp. 1380-1385, 2000.

[7] Jiřičková, M. \& Černý, R., Chloride Binding in Building Materials, Journal of Building Physics, 29, 189-200, 2006. 